\title{
Transitions through Critical Temperatures in Nematic
} Liquid Crystals

\author{
by
}

\author{
Apala Majumdar \\ John Ockendon \\ Peter Howell \\ Elena Surovyatkina
}





\title{
Transitions through Critical Temperatures in Nematic Liquid Crystals
}

\author{
Apala Majumdar, John Ockendon $†$ Peter Howell $\ddagger$ Elena Surovyatkina §
}

October 18, 2012

\begin{abstract}
We obtain 'dynamic' estimates for critical nematic liquid crystal (LC) temperatures with a slowly varying temperature-dependent control variable. We focus on two critical temperatures : the supercooling temperature below which the isotropic phase loses stability and the superheating temperature above which the ordered nematic states do not exist. In contrast to the static problem, the isotropic phase exhibits a memory effect below the supercooling temperature. This delayed loss of stability is independent of the rate of change of temperature and depends purely on the initial value of the temperature.
\end{abstract}

Keywords: Landau-de Gennes theory, order parameters, gradient-flow, time-dependent bifurcation parameters

\section{Introduction}

Nematic LCs are complex anisotropic liquids with a degree of long-range orientational ordering $[4,19]$. Of key importance is the concept of a 'scalar order parameter' that can distinguish between a disordered isotropic phase and an ordered nematic phase $[11,14]$. Order parameters are of prime importance in various branches of condensed matter physics e.g. superconductivity [3], active systems [7] and are used to describe transitions between different admissible equilibria. We focus on thermotropic LCs wherein the degree of orientational ordering is dictated by the temperature [4]. The isotropic-nematic phase transition has been extensively studied for thermotropic LCs [11, 15, 19], without paying attention to the effects of spatial

\footnotetext{
${ }^{*}$ Department of Mathematical Sciences, University of Bath, BA2 7AY (a.majumdar@bath.ac.uk, majumdar@maths.ox.ac.uk).

${ }^{\dagger}$ OCIAM, Mathematical Institute, University of Oxford.

${ }^{\ddagger}$ OCIAM, Mathematical Institute, University of Oxford.

${ }^{\S}$ Space Dynamics and Data Analysis Department, Space Research Institute, Russian Academy of Sciences, Moscow 117997, Russia
} 
and temporal variations in the temperature profile. In this paper, we study the non-equilibrium dynamics of the scalar order parameter when the temperature is a time-dependent control variable.

We work within the Landau-de Gennes ( $\mathrm{LdG}$ ) theory for thermotropic uniaxial nematic phases $[4,15,19]$. A uniaxial sample is characterized by two macroscopic variables : (i) the director, $\mathbf{n}$, which represents the preferred direction of molecular alignment and (ii) the order parameter, $S$, which is a measure of how well the molecules align with the director. In particular, the isotropic phase has identically vanishing order parameter, $S=0$, whereas a non-zero value of $S$ corresponds to an ordered nematic phase.

The LdG theory is a variational theory and equilibrium states correspond to minimizers of an appropriately defined LdG energy functional $[4,12,19]$. The isotropic-nematic phase transition is driven by the $\mathrm{LdG}$ thermotropic energy that contains a temperature-dependent parameter, denoted by $A$ throughout this paper; more details are given in Section 2 . The equilibrium order parameters at a given temperature, or equivalently for a given value of $A$, are given by the thermotropic energy minimizers [12]. There are two static critical values of $A$, associated with a bifurcation in the $(S, A)$-phase plane, in the LdG framework. The first critical temperature is the supercooling temperature below which the isotropic phase loses stability. The second critical temperature is the superheating temperature above which the nematic phases do not exist and the isotropic phase is the unique thermotropic energy minimizer. The supercooling and superheating temperatures are associated with a transcritical and a saddle-node bifurcation in the $(S, A)$-response diagram respectively, as illustrated in Figure 1.

The static critical temperatures are computed by treating the temperaturedependent variable, $A$, to be a constant. However, in many practical applications, $A$ is a function of a time and we study the evolution of the scalar order parameter, $S$, as $A$ slowly passes through the static critical values. We assume a simple gradient-flow model for the evolution of $S[11,15]$ and mathematically describe how the $(S, A)$-response diagram is modified by the slow time modulations of $A$. Our most striking result concerns the critical supercooling value. There is a marked difference between a monotonic increase and a monotonic decrease through this critical value which implies that experimental measurements can be sensitive to the direction of temperature change. More precisely, the unstable isotropic branch exhibits a memory effect in the low-temperature regime, far below the predicted static supercooling value, and this delayed loss of stability is also observed in boundary-value problems for the LC scalar order parameter. This is indeed our central result: critical LC temperatures can be strongly dependent on spatial and temporal variations in the temperature profile.

The study of bifurcations with a time-dependent bifurcation parameter has a long history; the reader is referred to $[1,2,6,8,10,17]$. In general, the phenomenon of dynamic stability exchange depends on multiple factors such 
as the nature of the bifurcation point, the initial value of the control variable and the presence of imperfections etc. Of particular importance is the rate at which the control variable crosses its critical value, which we assume to be slow compared to the response time of the sample. In Section 2, we review the LdG theory for uniaxial nematics and present the mathematical models. In Section 3, we study the dynamic exchange of stability between distinct static equilibria when the control variable, $A$, slowly varies through the static critical values. In Section 3.1, we derive rigorous estimates for the transcritical bifurcation associated with the supercooling value and in Section 3.2, we perform local asymptotic analysis around both the supercooling and superheating values to describe the passage from isotropic to nematic equilibria and vice-versa. In Section 4 , we discuss generalizations to boundary-value problems and outline directions for future work.

\section{The Model}

The LdG theory describes the state of a nematic LC by a physical state variable: the LdG Q-tensor defined in terms of anisotropic macroscopic quantities $[4,12,15]$. In the uniaxial phase, the $\mathbf{Q}$-tensor can be written as

$$
\mathbf{Q}=S\left(\mathbf{n} \otimes \mathbf{n}-\frac{\mathbf{I}}{3}\right)
$$

where $S$ and $\mathbf{n}$ have been defined above. We take $\mathbf{n} \in S^{2}$ to be a constant unit-vector throughout this paper, since we are interested in the spatiotemporal response of $S$ to temperature variations. This assumption is justified for samples with untreated boundaries wherein there are no external effects on the director field.

We work with a simple form of the LdG energy functional on a threedimensional (3D) domain, $\Omega \subset \mathbb{R}^{3}$, given by $[11,12]$

$$
I[\mathbf{Q}]:=\int_{\Omega} \frac{L}{2}|\nabla \mathbf{Q}|^{2}+f_{B}(\mathbf{Q}) d V
$$

where $L>0$ is an elastic constant and $f_{B}(\mathbf{Q})$ is the thermotropic energy density. The thermotropic energy contains quantitative information about the degree of orientational ordering and we work with the simplest, physically reasonable form of $f_{B}$ proposed in $[4,15]$, namely

$$
f_{B}(\mathbf{Q}):=\frac{A(T)}{2} \operatorname{tr} \mathbf{Q}^{2}-\frac{B}{3} \operatorname{tr} \mathbf{Q}^{3}+\frac{C}{4}\left(\operatorname{tr} \mathbf{Q}^{2}\right)^{2} .
$$

In (3), $A(T)$ is a linear function of the absolute temperature, $T$, given by $A(T)=\alpha\left(T-T^{*}\right)$ and $\alpha, B, C>0$ are positive material-dependent constants. In what follows, we refer to $A$ as temperature even though it is the re-scaled temperature. It can be explicitly shown that the stationary points 
of the quartic $f_{B}$ in (3) are either uniaxial or isotropic $[4,12,15,19]$. For a uniaxial $\mathbf{Q}$ (of the form (1)), $f_{B}(\mathbf{Q})$ reduces to a quartic polynomial in $S$,

$$
f_{B}(S)=\frac{A(T)}{3} S^{2}-\frac{2 B}{27} S^{3}+\frac{C}{9} S^{4}
$$

and the equilibrium order parameters, $S_{e q}(T)$, correspond to stationary points of this polynomial. For $A<\frac{B^{2}}{24 C}$, there are three distinct equilibria: (i) two ordered nematic branches

$$
\begin{array}{ll}
S_{+}(A)=\frac{B+\sqrt{B^{2}-24 A C}}{4 C} & \text { i.e. upper parabolic equilibrium(5) } \\
S_{-}(A)=\frac{B-\sqrt{B^{2}-24 A C}}{4 C} & \text { i.e. lower parabolic equilibrium (6) }
\end{array}
$$

and the isotropic branch, $S=0$. For $A>\frac{B^{2}}{24 C}$, the ordered nematic states do not exist and the isotropic branch is the unique thermotropic energy minimizer.

The supercooling temperature corresponds to $A=0$ and is associated with an exchange of stability between $S_{-}(A)$ and $S=0$. On the one hand, $S=0$ is stable for $A>0$ and unstable for $A<0$ and on the other hand, $S_{-}(A)$ is locally stable for $A<0$ and unstable for $0<A<\frac{B^{2}}{24 C}$. The superheating temperature is denoted by $A_{J}=\frac{B^{2}}{24 C} ; A=A_{J}$ is associated with a saddle-node bifurcation such that the two nematic equilibria, $S_{ \pm}(A)$, coalesce at $A_{J}$ and cease to exist for $A>A_{J}$.

As in $[11,15]$, we assume a simple gradient-flow model to describe the evolution of the scalar order parameter, as shown below :

$$
\frac{\partial S}{\partial \bar{t}}=\frac{2 \bar{L}}{3} \bar{\nabla}^{2} S-\frac{2 S}{9}\left(2 \bar{C} S^{2}-\bar{B} S+3 \bar{A}(T(\mathbf{x}, t))\right) .
$$

The model (7) has been non-dimensionalized using the scalings, $\overline{\mathbf{x}}=\frac{\mathbf{x}}{D}$ and $\bar{t}=\frac{A_{*}}{\gamma} t$, where $D$ is a characteristic domain length, $\gamma$ is the viscosity coefficient and $A_{*}$ is an arbitrary characteristic value of $A$ in (4). The rescaled dimensionless variables are related to the original variables by $\bar{L}=$ $\frac{L}{A_{*} D^{2}}, \bar{B}=\frac{B}{A_{*}}, \bar{C}=\frac{C}{A_{*}}, \bar{A}=\frac{A}{A_{*}}$. We assume that $\bar{B}$ and $\bar{C}$ are positive constants of comparable magnitude. The model (7) is complemented by suitably defined initial and boundary conditions. In most of what follows, we study the simpler spatially homogeneous case wherein the model (7) reduces to an ordinary differential equation with a slowly varying time-dependent bifurcation parameter, $A(T(t))$ :

$$
\begin{aligned}
& \frac{d S}{d \bar{t}}=-\frac{2 S}{9}\left(2 \bar{C} S^{2}-\bar{B} S+3 \bar{A}(T(t))\right) \quad S\left(\bar{t}_{0}\right)=S_{0}, \\
& \frac{d \bar{A}}{d \bar{t}}=\epsilon \operatorname{sgn} \epsilon \quad \bar{A}\left(\bar{t}_{0}\right)=\bar{A}_{0} .
\end{aligned}
$$


In (9), $0<\epsilon<1$ is a small parameter controlling the rate of change of temperature and we take $\epsilon \ll \min \left\{\bar{B}, \bar{C}, \frac{\gamma}{A_{*}^{2}}\right\}$. We drop the bars from the re-scaled variables in the following sections and all subsequent results are to be understood in terms of the dimensionless variables.

\section{Slow passage through critical values}

In the case of constant (time-independent) $A=A_{0}$, the ODE (8) has a unique monotonic solution $S\left(t, A_{0}\right)$, provided that $S_{0} \neq 0$ and $S_{0} \neq S_{ \pm}\left(A_{0}\right)$ where $S_{ \pm}$have been defined in (5)-(6). One can check that $S\left(t, A_{0}\right)$ is given by

$$
\begin{aligned}
& \exp \left[-\frac{4 C}{9}\left(t-t_{0}\right)\right]= \\
& =\left(\frac{S}{S_{0}}\right)^{3 C / 2 A}\left(\frac{S-S_{+}\left(A_{0}\right)}{S_{0}-S_{+}\left(A_{0}\right)}\right)^{\frac{1}{S_{+}\left(S_{+}-S_{-}\right)}}\left(\frac{S_{0}-S_{-}\left(A_{0}\right)}{S-S_{-}\left(A_{0}\right)}\right)^{\frac{1}{S_{-}\left(S_{+}-S_{-}\right.}(10)}
\end{aligned}
$$

Mostly, we consider initial conditions, $S_{0}$, within an $\epsilon$-neighbourhood of one of the stable equilibria at, $A=A_{0}$, since the system is likely to be near equilibrium when the process of temperature-change is initiated.

We cannot compute explicit analytic solutions for the fully time-dependent problem (8)-(9). However, we can make the following qualitative observations: (i) the parabolic equilibria (see (5)-(6)) partition the $(S, A)$-plane into monotonically increasing $\left(\frac{d S}{d t}>0\right)$ and monotonically decreasing $\left(\frac{d S}{d t}<0\right)$ sections as illustrated in Figure 1 and (ii) the solutions do not cross the isotropic branch, $S=0$, as can be seen from a straightforward analysis of (8). These two properties collectively determine the qualitative solution properties, subject to arbitrary initial conditions. In the following subsections, we study the slow passage through the static critical values, $A=0$ and $A=A_{J}$, and quantitatively describe the delayed transitions between different equilibria.

\subsection{Estimates for the transcritical bifurcation at $A=0$}

The slow passage through a transcritical bifurcation point has been studied in detail in [10]. For completeness, we recall the main result in [10] below.

Theorem 1. [10] Consider the initial-value problem

$$
\begin{aligned}
& \frac{d x}{d t}=\epsilon f(x, y) \quad x(0)=a \\
& \frac{d y}{d t}=g(x, y) \quad y(0)=b .
\end{aligned}
$$

Suppose further that $g(x, \phi(x))=g(x, \psi(x))=0$ for continuously differentiable functions $\phi(x), \psi(x)$. The point $x=c$ is a bifurcation point such that 
$\phi(c)=\psi(c), \phi(x)$ is stable for $x<c$ and unstable otherwise and $\psi(x)$ is unstable for $x<c$ and stable otherwise. Define the composed solution

$$
Y(t)= \begin{cases}\phi(x(t)) & x \leq c \\ \psi(x(t)) & x>c .\end{cases}
$$

Let $\alpha=\left.\frac{\partial \phi}{\partial x}\right|_{x=c}$ and assume that

$$
\alpha>0
$$

Then there exist $a\langle c, b\rangle c, C^{*}>0$ and $\epsilon^{*}>0$ such that the solution of (11) exists on $[a, b]$ and satisfies

$$
|y(t, \epsilon)-Y(t)| \leq C^{*} \sqrt{\epsilon} \quad t \in[a, b] ; 0<\epsilon<\epsilon^{*} .
$$

As we shall see, Theorem 1 only captures the forward transition through $A=0$ i.e. when $A$ monotonically increases through the supercooling value and not the backward transition. Let

$$
A(t)=A_{0}+\epsilon t
$$

where $t \geq 0, A_{0}<0$ and $A_{0}$ is independent of $\epsilon$ i.e. the solution branch starts bounded away from the critical value, $A=0$. The two competing equilibria near $A=0$ are the lower parabolic equilibrium, $S_{-}(A)$ in (5), and the isotropic branch, $S=0$. For small $A, S_{-}(A)$ can be approximated by

$$
S_{-}(A) \sim \frac{3 A}{B}
$$

and $\frac{d S_{-}}{d A}>0$ at $A=0$. In the forward case, $S_{-}(A)$ plays the role of $\phi$ in (12) and therefore, $\alpha=\frac{d S_{-}}{d A}>0$ and the hypothesis of Theorem 1 is satisfied. We deduce that the full solution, $S\left(t, A_{0}, S_{0}, \epsilon\right)$, remains in an $\epsilon$-neighbourhood of $S_{-}(A(t))$ over the interval $A \in\left[A_{0}, a\right]$ for some $a<0$ with $|a| \ll 1$, followed by a transitional regime within which $S$ is of $O(\sqrt{\epsilon})$, after which $S$ moves into an $\epsilon$-neighbourhood of the isotropic branch for $A \in[b, \infty)$, with $0<b \ll 1$.

We can glean the leading order behaviour of $\frac{S}{\sqrt{\epsilon}}$ from explicit lower and upper solutions for $S\left(t, A_{0}, S_{0}, \epsilon\right)$, as shown below; see [16] for similar examples.

$$
-\sqrt{\epsilon} \exp \left[-\frac{A^{2}}{3 \epsilon}\right] \leq S\left(t, A_{0}, S_{0}, \epsilon\right) \leq-\frac{9 \epsilon}{4 B} \frac{\exp \left[-\frac{A^{2}}{3 \epsilon}\right]}{\int_{-\infty}^{A} \exp \left[-\eta^{2} / 3 \epsilon\right] d \eta}
$$

Therefore, following a transition within an $O(\sqrt{\epsilon})$-neighbourhood of $A=0$, the solution is exponentially small in $\epsilon$. In Figure 2, we numerically solve 
the system (8)-(9) for the case of a slow monotonic increase through $A=0$, with different values of $\epsilon$. The solution migrates to an $\epsilon$-neighbourhood of $S=0$ within a temperature range of width $O(\sqrt{-\epsilon \ln \epsilon})$ around $A=0$ and this is in agreement with the upper and lower solutions in (15).

The next step is to consider the backward transition through $A=0$. Let

$$
A(t)=A_{0}-\epsilon t,
$$

with $A_{0}>0$ independent of $\epsilon$, initial condition $S_{0}=\epsilon \beta>0$ and $\beta$ independent of $\epsilon$. Analogous arguments apply to the case $S_{0}<0$. We observe that the backward transition does not satisfy the hypotheses of Theorem 1 , since in the backward case, the isotropic branch is analogous to $\phi$ in (12). Therefore, $\alpha=0$ in Theorem 1 and we do not expect an immediate transition from the isotropic branch to the parabolic equilibria for $A<0$. In the following proposition, we demonstrate that the unstable isotropic phase persists over the interval $A \in\left(-A_{0}, 0\right)$ i.e. the delay in stability exchange is independent of $\epsilon$ and is proportional to the initial temperature. We follow the paradigm in [18] and reproduce the necessary details for completeness.

Proposition 1. Let $S\left(t, A_{0}, S_{0}, \epsilon\right)$ denote a solution of the initial-value problem (8)-(9), with $A(t)=A_{0}-\epsilon t$ and $S_{0}=\beta \epsilon>0$. For $\epsilon>0$ sufficiently small and for $A_{0}>0$ independent of $\epsilon$, we have

$$
0<S\left(t, A_{0}, S_{0}, \epsilon\right) \leq \beta \epsilon
$$

for $A \in\left[-A_{0}, A_{0}\right]$.

Proof: From qualitative solution properties (see Figure 1), the solution $S\left(t, A_{0}, S_{0}, \epsilon\right)$ descends towards the isotropic branch for $A \in\left(0, A_{0}\right]$, followed by a monotonic increase for $A<0$.

One can verify that there exists a positive constant $\alpha>0$, independent of $\epsilon$, such that

$$
\left(-\frac{2 A}{3}-\alpha\right) S \leq \frac{d S}{d t} \leq\left(-\frac{2 A}{3}+\alpha\right) S
$$

for $S \in(0, \beta \epsilon)$ and $A \in\left(-2 A_{0}, 2 A_{0}\right)$. The ordinary differential equations

$$
\frac{d S}{d t}=\left(-\frac{2 A}{3} \pm \alpha\right) S
$$

can be solved explicitly with $A(t)=A_{0}-\epsilon t$ and initial condition, $S_{0}=\beta \epsilon$. The corresponding solutions are, $S^{ \pm}\left(t, A_{0}, \epsilon\right)$, where

$$
\ln \left(\frac{S^{ \pm}}{\beta \epsilon}\right)=\frac{1}{3 \epsilon}\left[A^{2}-A_{0}^{2} \pm 3 \alpha\left(A-A_{0}\right)\right] .
$$


The upper curve, $S^{+}$, re-intersects the line, $S=\beta \epsilon$, again at

$$
A_{+}=-\frac{3 \alpha+\sqrt{9 \alpha^{2}+4 A_{0}^{2}+12 \alpha A_{0}}}{2}
$$

and the lower curve, $S^{-}$, re-intersects the line, $S=\beta \epsilon$, again at

$$
A_{-}=\frac{3 \alpha-\sqrt{9 \alpha^{2}+4 A_{0}^{2}-12 \alpha A_{0}}}{2} .
$$

Let $A_{*}\left(A_{0}, \epsilon\right)$ denote the first point of re-intersection between the solution, $S\left(t, A_{0}, S_{0}, \epsilon\right)$, and the line, $S=\beta \epsilon$. From the inequalities (18) and the values $A_{ \pm}$above, we deduce that $A_{+} \leq A_{*}\left(A_{0}, \epsilon\right) \leq A_{-}$. These estimates are true for any $0<\alpha \leq \frac{A_{0}}{3}$, independent of $\epsilon$. For a fixed $\epsilon>0$, we take the limit $\alpha \rightarrow 0$ to deduce that

$$
A_{*}\left(A_{0}, \epsilon\right)=-A_{0}<0 .
$$

The conclusion of Proposition 1 now follows.

\subsection{Asymptotic analysis for small $\epsilon$.}

We re-scale (8)-(9) according to $\tilde{S}=\frac{4 C}{B} S, \tau=\epsilon \frac{B^{2}}{36 C} t$ so that (8) becomes

$$
\epsilon \frac{d \tilde{S}}{d \tau}=-\tilde{S}\left[\tilde{S}^{2}-2 \tilde{S}+\tilde{A}(\tau)\right] \quad \tilde{S}(0)=\tilde{S}_{0}
$$

where

$$
\tilde{A}(\tau)=\frac{24 A(t) C}{B^{2}} .
$$

The form (22) is more amenable to asymptotic analysis. The static critical values are $\tilde{A}=0$ (the supercooling value) and $\tilde{A}=+1$ (the superheating value) respectively, and the parabolic equilibria are given by, $\tilde{S}_{ \pm}=1 \pm$ $\sqrt{1-\tilde{A}}$ for $\tilde{A} \leq 1$.

\subsubsection{Forward transition through $\tilde{A}=0$}

Let $\tilde{A}(\tau)=A_{0}+\tau$ for some $A_{0}<0$ and the corresponding initial condition, $\tilde{S}_{0}<0$, is within an $\epsilon$-neighbourhood of the lower parabolic equilibrium $\tilde{S}_{-}\left(A_{0}\right)$. For an arbitrary initial condition $\tilde{S}_{0}<0$, the solution migrates to an $\epsilon$-neighbourhood of the lower parabolic branch, $\tilde{S}_{-}(\tilde{A})$, over a temperature range of width $O(\epsilon)$ around the initial value, $\tilde{A}=A_{0}$. We have already seen in Theorem 1 that the solution remains within an $\epsilon$-neighbourhood of $\tilde{S}_{-}(\tilde{A})$ for $|\tilde{A}| \gg \sqrt{\epsilon}$; also see [8] for generic asymptotic methods for first-order bifurcation problems. 
In conformity with Theorem 1, we introduce the scalings, $\tilde{S}=\sqrt{\epsilon} \tilde{S}^{\prime}$ and $\tau=-A_{0}+\sqrt{\epsilon} \tau^{\prime}$, near the critical value, $\tilde{A}=0$. The leading-order problem is an exactly solvable Bernoulli ODE

$$
\frac{d \tilde{S}^{\prime}}{d \tau^{\prime}}=2 \tilde{S}^{\prime}\left(\tilde{S}^{\prime}-\frac{\tau^{\prime}}{2}\right)
$$

The matching condition that requires the solution to approach $\tilde{S}_{-}(\tilde{A})$ as $\tau^{\prime} \rightarrow-\infty$, is $\tilde{S}^{\prime}\left(\tau^{\prime}\right) \rightarrow \frac{\tau^{\prime}}{2}$ as $\tau^{\prime} \rightarrow-\infty$. This problem has a unique solution as shown below:

$$
\tilde{S}^{\prime}\left(\tau^{\prime}\right)=-\frac{\exp \left[-\left(\tau^{\prime}\right)^{2} / 2\right]}{2 \int_{-\infty}^{\tau^{\prime}} \exp \left[-\eta^{2} / 2\right] d \eta}
$$

which demonstrates the smooth approach to the isotropic branch from below, as $\tau \rightarrow+\infty$. The solution (24) is consistent with the bounds in (15) and yields a sharper estimate for the solution in the transition layer.

\subsubsection{Backward transition through $\tilde{A}=0$}

We take $\tilde{A}(\tau)=A_{0}-\tau$ for some $0<A_{0}<1$ and the initial condition, $\tilde{S}_{0}$, to be within the basin of attraction of the isotropic equilibrium at $A=A_{0}$. This is in contrast to Proposition 1 where we only consider initial conditions within an $\epsilon$-neighbourhood of the isotropic branch.

From the $(S, A)$-phase plane, the solution descends towards the isotropic branch, in a layer of width $O(\epsilon)$, for $\tilde{A}>0$ and the leading order equation in this region is

$$
\frac{d \tilde{S}}{d \tau^{\prime}}=-\tilde{S}\left[\tilde{S}^{2}-2 \tilde{S}+A_{0}\right]
$$

where $\tau^{\prime}=\frac{\tau}{\epsilon}$. The leading-order solution is implicitly given by

$$
\tau^{\prime}=\frac{\log \left(\frac{\tilde{S}_{0}^{2}\left(A_{0}+\tilde{S}^{2}-2 \tilde{S}\right)}{\tilde{S}^{2}\left(A_{0}+\tilde{S}_{0}^{2}-2 \tilde{S}_{0}\right)}\right)}{2 A_{0}}+\frac{\tanh ^{-1}\left(\frac{\sqrt{1-A_{0}}\left(\tilde{S}_{0}-\tilde{S}\right)}{A_{0}-\tilde{S}+(\tilde{S}-1) \tilde{S}_{0}}\right)}{A_{0} \sqrt{1-A_{0}}} .
$$

As $\tau^{\prime} \rightarrow+\infty$, we have $\tilde{S} \sim B_{0} \exp \left[-A_{0} \tau^{\prime}\right]$ where

$$
\log \left(B_{0}\right)=\frac{1}{2} \log \left(\frac{A_{0} \tilde{S}_{0}^{2}}{\tilde{S}_{0}^{2}-2 \tilde{S}_{0}+A_{0}}\right)+\frac{\tanh ^{-1}\left(\frac{\sqrt{1-A_{0}} \tilde{S}_{0}}{A_{0}-\tilde{S}_{0}}\right)}{\sqrt{1-A_{0}}} .
$$

Thereafter, $\tilde{S}$ becomes small for $\tilde{A}<A_{0}$ and the subsequent behaviour may be found by linearizing (22) about $\tilde{S}=0$,

$$
\epsilon \frac{d \tilde{S}}{d \tau}+\left(A_{0}-\tau\right) \tilde{S}=0,
$$


with matching condition, $\tilde{S} \sim B_{0} \exp \left[-A_{0} \frac{\tau}{\epsilon}\right]$, as $\tau \rightarrow 0^{+}$. The leading-order solution for $\tau>0$ is given by

$$
\tilde{S}(\tau) \sim B_{0} \exp \left[\frac{-A_{0} \tau+\frac{\tau^{2}}{2}}{\epsilon}\right]
$$

which is exponentially small for $0<\tau<2 A_{0}$, or for $A \in\left(-A_{0}, A_{0}\right)$, and is consistent with the lower and upper solutions computed in (20).

Near $\tilde{A}=-A_{0}$, we set $\tau=2 A_{0}+\epsilon \tau^{\prime}$ to get the leading order equation

$$
\frac{d \tilde{S}}{d \tau^{\prime}}=-\tilde{S}\left(\tilde{S}^{2}-2 \tilde{S}-A_{0}\right)
$$

with matching condition, $\tilde{S}\left(\tau^{\prime}\right) \rightarrow B_{0} \exp \left[+A_{0} \tau^{\prime}\right]$ as $\tau^{\prime} \rightarrow-\infty$. As in (26), we obtain

$$
\tau^{\prime}=-\frac{\log \left(\frac{B_{0}^{2}\left(A_{0}-\tilde{S}^{2}+2 \tilde{S}\right)}{A_{0} \tilde{S}^{2}}\right)}{2 A_{0}}-\frac{\tanh ^{-1}\left(\frac{\left.\sqrt{1+A_{0}} \tilde{S}\right)}{A_{0}+\tilde{S}}\right)}{A_{0} \sqrt{1+A_{0}}},
$$

where $B_{0}$ has been defined in (27) and this describes the isotropic-upper parabolic ascent, in a layer of width $O(\epsilon)$, for $\tilde{A}<0$.

If the initial condition is $\tilde{S}_{0}<0$, then we can repeat the computations above to describe the transition from the isotropic branch to the lower parabolic branch in (6) for $\tilde{A}<0$. This asymptotic analysis shows that the delay phenomenon is characteristic of any initial condition, $\tilde{S}_{0}$, within the basin of attraction of $\tilde{S}=0$. The delay itself is independent of $\tilde{S}_{0}$ but $\tilde{S}_{0}$ manifests in the final isotropic-parabolic approach in (30).

\subsubsection{Forward transition through $\tilde{A}=1$.}

Let $\tilde{A}(\tau)=A_{0}+\tau$ for some $A_{0}<1$ and let the initial condition, $\tilde{S}_{0}$, be within an $\epsilon$-neighourhood of the upper parabolic branch, $\tilde{S}_{+}\left(A_{0}\right)$. It can be rigorously proven that the solution remains within an $\epsilon$-neighbourhood of $\tilde{S}_{+}(\tilde{A})$ provided that $\tilde{A}$ is bounded away from $\tilde{A}=1[1,17]$. Indeed, the solution may be expanded asymptotically in the form

$$
\tilde{S} \sim 1+\sqrt{1-\tilde{A}}+\frac{\epsilon}{4(1-\tilde{A})(1+\sqrt{1-\tilde{A}})}+O\left(\epsilon^{2}\right)
$$

and we infer that there is a nonuniformity when $1-\tilde{A}=O\left(\epsilon^{2 / 3}\right)$ and $\tilde{S}-1=O\left(\epsilon^{1 / 3}\right)$. We, therefore, define $\bar{\tau}=\epsilon^{-2 / 3}\left(1-A_{0}-\tau\right)$, and seek an asymptotic expansion for $\tilde{S}(\bar{\tau})$ from $(22)$ as follows:

$$
\tilde{S}(\bar{\tau})=1+\epsilon^{1 / 3} S_{1}(\bar{\tau})+\epsilon^{2 / 3} S_{2}(\bar{\tau})+\ldots
$$


The first-order term is a solution of the Riccati equation

$$
\frac{d S_{1}}{d \bar{\tau}}=-\left(S_{1}^{2}+\bar{\tau}^{2}\right)
$$

and therefore,

$$
\begin{aligned}
& S_{1}(\bar{\tau})=-\frac{\operatorname{Ai}^{\prime}(-\bar{\tau})}{\operatorname{Ai}(-\bar{\tau})}, \\
& S_{2}(\bar{\tau})=\frac{\int_{-\bar{\tau}}^{\infty} \operatorname{Ai}^{\prime}(z)^{3} / \operatorname{Ai}(z) d z}{\operatorname{Ai}(-\bar{\tau})^{2}}+\frac{\operatorname{Ai}^{\prime}(-\bar{\tau})^{2}}{2 \operatorname{Ai}(-\bar{\tau})^{2}},
\end{aligned}
$$

where Ai denotes the Airy function [8].

This expansion becomes nonuniform as $\bar{\tau} \rightarrow \tau^{*}$ where $-\tau^{*}$ denotes the first zero of the Airy Function i.e. $\tau^{*}=+2.338$. In this limit,

$$
\tilde{S}(\bar{\tau}) \sim 1-\frac{\epsilon^{1 / 3}}{\tau^{*}-\bar{\tau}}-\epsilon^{2 / 3} \frac{C_{1}+\log \left(\tau^{*}-\bar{\tau}\right)}{\left(\tau^{*}-\bar{\tau}\right)^{2}}+\ldots
$$

where $C_{1} \approx 0.5085$ is an explicitly computable positive constant. This motivates the scaling, $\tilde{A}=1+\tau^{*} \epsilon^{2 / 3}+\frac{1}{3} \epsilon \log \left(\frac{1}{\epsilon}\right)+\epsilon \tilde{\tau}$, which results in the leading order equation (35) below,

$$
\frac{d \tilde{S}}{d \tilde{\tau}} \sim-\tilde{S}(\tilde{S}-1)^{2}
$$

with matching condition

$$
\tilde{S} \sim 1+\frac{1}{\tilde{\tau}}-\frac{C_{1}+\log (-\tilde{\tau})}{\tilde{\tau}^{2}}+\ldots
$$

as $\tilde{\tau} \rightarrow-\infty$. The leading-order inner solution is then implicitly given by

$$
\tilde{\tau}=-C_{1}-\frac{1}{1-\tilde{S}}+\log \left(\frac{1-\tilde{S}}{\tilde{S}}\right) .
$$

Thus, the temperature overshoots the critical value $\tilde{A}=1$ by a distance $\Delta \tilde{A} \sim \tau^{*} \epsilon^{2 / 3}+\frac{1}{3} \epsilon \log \left(\frac{1}{\epsilon}\right)$ before transitioning down to the isotropic branch over a temperature range of order $\epsilon$.

\section{Conclusion and Discussion}

This paper focuses on delay estimates for the exchange of stability across bifurcation points in thermotropic uniaxial nematics, with a slowly varying control variable. We have adopted a simple gradient-flow model to describe the evolution of the nematic scalar order parameter in space and time, and in the simple case of a spatially homogeneous system, our model falls within the 
well-studied framework of first-order algebraic bifurcation problems. With reference to Figure 1, we are primarily interested in two critical values : (i) the supercooling value, $A=0$ and (ii) the superheating value, $A_{J}=\frac{B^{2}}{24 C}$. The superheating value corresponds to a saddle-node bifurcation and, $A(\epsilon t)$ with $0<\epsilon \ll 1$, regularizes the exchange of stability between the ordered nematic equilibria and the isotropic branch over a region of width, $O\left(\epsilon^{2 / 3}\right)$, centered around $A=A_{J}$. In this case, we do not expect any marked changes in macroscopic measurements of $A_{J}$.

Our most striking observation concerns the supercooling value, $A=0$. In the forward transition case (see Sections 3.1 and 3.2.1), the exchange of stability takes place within a region of width, $O(\sqrt{\epsilon})$, centered around $A=0$ and we recover the static value in the limit $\epsilon \rightarrow 0$. In the backward case (see Sections 3.1 and 3.2.2), the exchange of stability takes place within a region of width, $O\left(2 A_{0}\right)$, around $A=0$, where $A_{0}$ is the initial temperature. In particular, we do not recover the static value in the limit $\epsilon \rightarrow 0$.

The results in Section 3 are restricted to spatially homogeneous systems. Some of our results can be easily generalized to inhomogeneous systems. As an illustrative example, consider the partial differential equation (7) on a one-dimensional (re-scaled) interval, $0 \leq \bar{x} \leq 1$, as shown below

$$
\frac{\partial S}{\partial \bar{t}}=\frac{2 \bar{L}}{3} \frac{\partial^{2} S}{\partial \bar{x}^{2}}-\frac{2 S}{9}\left(2 \bar{C} S^{2}-\bar{B} S+3 \bar{A}(\bar{x}, t)\right) \quad 0 \leq \bar{x} \leq 1, \bar{t} \geq 0 .
$$

Recall that $\bar{t}, \bar{L}$ etc. are dimensionless and related to the original variables by the re-scalings introduced in Section 2. For simplicity, we impose homogeneous boundary conditions, $S(0, \bar{t})=S(1, \bar{t})=0$. The initial condition can be any arbitrary function 'sufficiently close' to the isotropic equilibrium. We study the nonlinear 'static' stability of the isotropic equilibrium by computing the second variation of the LdG energy in (2), the positivity of which is a sufficient criterion for nonlinear 'static' stability in one spatial dimension $[5,13]$. The second variation of the associated dimensionless LdG energy about $S=0$ is given by

$$
\left.\delta^{2} I\right|_{S=0}=\int_{0}^{1} \frac{2}{3} \bar{L}\left(\frac{d \theta}{d \bar{x}}\right)^{2}+\bar{A} \theta^{2}(\bar{x}) d \bar{x},
$$

where $\theta$ is an arbitrary continuously differentiable function vanishing at the boundary. Recalling Wirtinger's inequality [13] i.e.

$$
\int_{0}^{1}\left(\frac{d \theta}{d \bar{x}}\right)^{2} d \bar{x} \geq \pi^{2} \int_{0}^{1} \theta^{2}(\bar{x}) d \bar{x}
$$

for all such $\theta$, we obtain the following inequality :

$$
\left.\delta^{2} I\right|_{S=0} \geq \frac{2}{3} \int_{0}^{1}\left(\bar{L} \pi^{2}+\bar{A}\right) \theta^{2}(\bar{x}) d \bar{x}
$$


and one can immediately see that $\left.\delta^{2} I\right|_{S=0}>0$ if

$$
\min _{\bar{x} \in[0,1] ; \bar{t} \geq 0} \bar{A}(\bar{x}, \bar{t})>\bar{A}_{T}=-\pi^{2} \bar{L}
$$

or in terms of the original variables, the isotropic state is stable (in the static sense) if

$$
\min _{x \in[0, D] ; t \geq 0} A(x, t)>A_{T}=-\pi^{2} \frac{L}{D^{2}} .
$$

In (42), $L$ is an elastic constant and $D$ is the interval length. Thus, the isotropic equilibrium is stable for $A>A_{T}$ and $A_{T}$ is strictly less than the homogeneous supercooling value, $A=0$. Having demonstrated the nonlinear 'static stability' of the isotropic phase for $A>A_{T}$, we can use Liapounov's direct method (with the LdG energy as a Liapounov function) to prove the dynamic Liapounov stability of the isotropic phase for $A>A_{T}$ [5]. This delayed loss of stability is purely a consequence of the boundary effects.

The preceding discussion shows that measurements of the supercooling temperature can be sensitive to spatial and temporal variations in the temperature profile. From an applications perspective, one needs to have reliable estimates for the temperature range over which an ordered LC phase can be observed [4]. These delay estimates imply the persistence of a disordered, isotropic phase for low temperatures and this persistence will affect the properties of LC-based devices. Of course, the evolution of the LC phase with temperature depends on a combination of factors, not all of which are included in our simplistic model. For example, we assume a constant director field $\mathbf{n}$ in (4) and in practice, $\mathbf{n}$, surface anchoring and elastic anisotropy effects couple to the LC scalar order parameter. Preliminary investigations suggest that noise can play a crucial role in stability exchange phenomena and there is a delicate balance between the relative magnitudes of noise and $\epsilon$ in our model. Nevertheless, although our model represents an idealized situation, it draws attention to a hitherto neglected effect : the sensitivity of critical LC temperatures to the temperature distribution itself, and we plan to incorporate additional effects in future work.

Acknowledgements: AM is supported by an EPSRC Career Acceleration Fellowship EP/J001686/1 and a Keble Research Fellowship, Keble College, University of Oxford. This publication is partly based on work supported by Award No. KUK-C1-013-04, made by King Abdullah University of Science and Technology (KAUST). AM would like to thank the Oxford Centre for Collaborative Applied Mathematics (OCCAM) for its hospitality over the period August - October 2012. ES would like to thank OCCAM for funding a collaborative visit in 2009. JRO gratefully acknowledges receipt of a Leverhulme Emeritus Fellowship while this paper was being completed. 


\section{References}

[1] C.Baesens, Slow sweep through a period-doubling cascade: Delayed bifurcations and renormalisation. Physica D: Nonlinear Phenomena, 53, $24,319-375$ (1991).

[2] I.R.Collinge and J.R.Ockendon, Transition Through Resonance of a Duffing Oscillator. SIAM J. Appl. Math., 37(2), 350357 (1979).

[3] S.J.Chapman, S.D.Howison and J.R.Ockendon, Macroscopic Models of Superconductivity. SIAM Review 34, 529-560 (1992).

[4] P.G.De Gennes, The physics of liquid crystals. Oxford, Clarendon Press, 1974.

[5] R.E.Caflisch and J.H.Maddocks, Nonlinear dynamical theory of the elastica. Proceedings of the Royal Society of Edinburgh 99A, 1-23 (1984).

[6] T.Erneux and P.Mandel, Imperfect bifurcation with a slowly-varying control parameter. SIAM Journal on Applied Mathematics, 46, 1-15 (1986).

[7] L.Giomi, L.Mahadevan, B.Chakraborty and M.F.Hagan, Excitable Patterns in Active Nematics. Physical Review Letters 106, 218101-218104 (2011).

[8] R.Haberman, Slowly varying jump and transition phenomena associated with algebraic bifurcation problems. SIAM Journal on Applied Mathematics, 37, 69-106 (1979).

[9] A.Kapila, Arrhenius Systems: Dynamics of Jump Due to Slow Passage Through Criticality. SIAM Journal on Applied Mathematics, 41, 29-42 (1981).

[10] N.Lebovitz and R.Schaar, Exchange of stabilities in autonomous systems. Studies in Applied Mathematics, 54, 229-260 (1975).

[11] F.H.Lin and C.Liu, Static and Dynamic Theories of Liquid Crystals. Journal of Partial Differential Equations, 14, no. 4, 289-330 (2001).

[12] A.Majumdar, Equilibrium order parameters of liquid crystals in the Landau-de Gennes theory. European Journal of Applied Mathematics, 21, 181-203 (2010).

[13] A.Majumdar, C.Prior and A.Goriely, Stability estimates for a twisted rod under terminal loads: a 3D study. Journal of Elasticity, 109, Issue $1,75-93(2012)$. 
[14] N.D.Mermin, The topological theory of defects in ordered media. Reviews of Modern Physics, 51, 591-648 (1979).

[15] N.J.Mottram and C.Newton, Introduction to Q-tensor Theory. University of Strathclyde, Department of Mathematics, Research Report, 10 (2004).

[16] N.N.Nefedov and K.R.Schneider, Immediate exchange of stabilities in singularly perturbed systems. Differential and Integral Equations, 12, 583599 (1999).

[17] A.Neishtadt, On stability loss delay for dynamical bifurcations. I, II. Differential Equations, 23, 1385-1391 (1987); 24, 171-176 (1988).

[18] S.Schecter, Persistent unstable equilibria and closed orbits of a singularly perturbed equation. Journal of Differential Equations, 60, 131-141 (1985).

[19] E.G.Virga, Variational theories for liquid crystals. Chapman and Hall, London, 1994. 


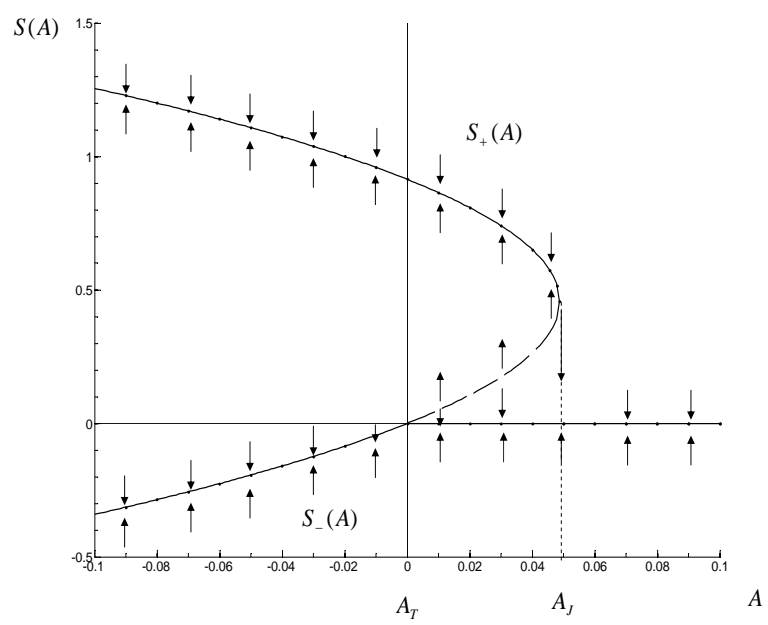

Figure 1: The $(S, A)$-phase plane with $B=0.64, C=0.35$, in dimensionless units [15]. Then $A_{J}=0.05$. The upward arrows indicate the direction of increasing $S$ i.e. where $\frac{d S}{d t}>0$ from (8)-(9), and the downward arrows indicate the direction of decreasing $S$ where $\frac{d S}{d t}<0$. 


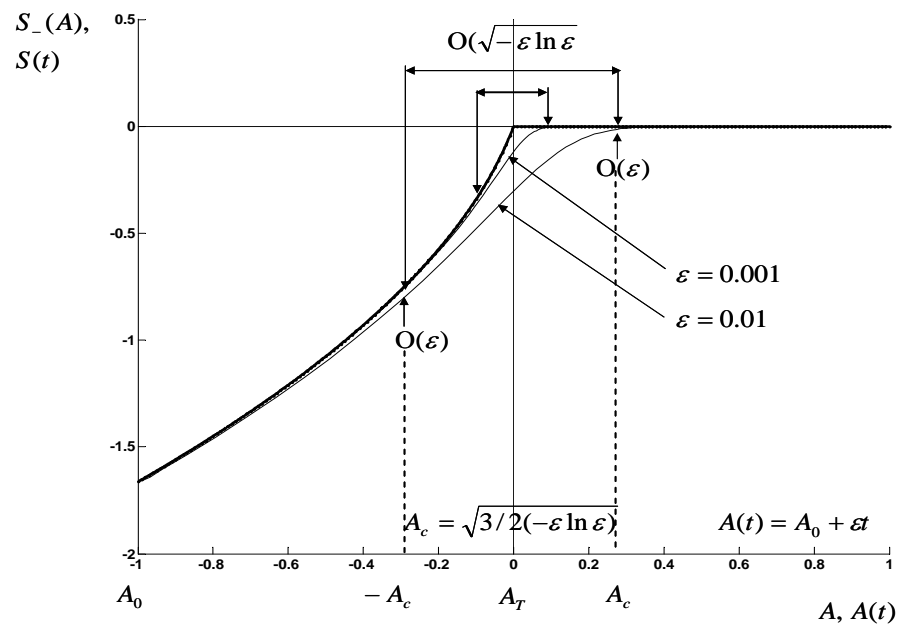

Figure 2: Numerical solutions of (8)-(9) with $A(t)=-1+\epsilon t$ and two different values of $\epsilon$ : (i) $\epsilon=0.01$ and (ii) $\epsilon=0.001$. We compute the width of the temperature band outside which $S\left(t, A_{0}, S_{0}, \epsilon\right)$ is within an $\epsilon$-neighbourhood of either the lower parabolic branch or the isotropic branch. This width is proportional to $O(\sqrt{-\epsilon \ln \epsilon})$, as can be seen by computing the value of $A$ for which the lower and upper solutions (see (15)) cross $S=-\epsilon$. 


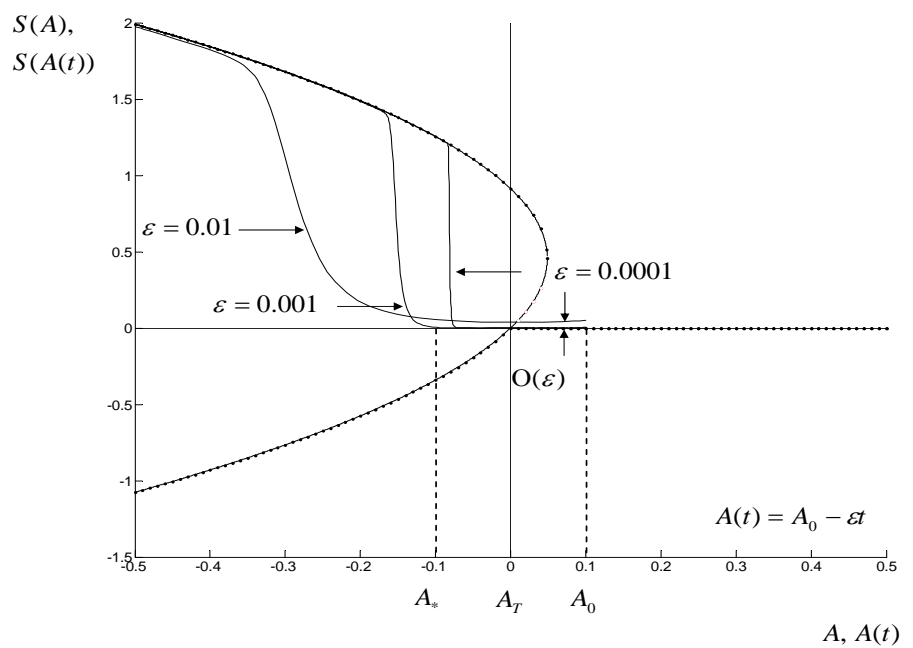

Figure 3: Numerical solutions of (8)-(9) with $A(t)=0.1-\epsilon t$, three different values of $\epsilon$ : (i) $\epsilon=0.01$, (ii) $\epsilon=0.001$ and (iii) $\epsilon=0.0001$ and initial condition $S_{0}=5 \epsilon$. 


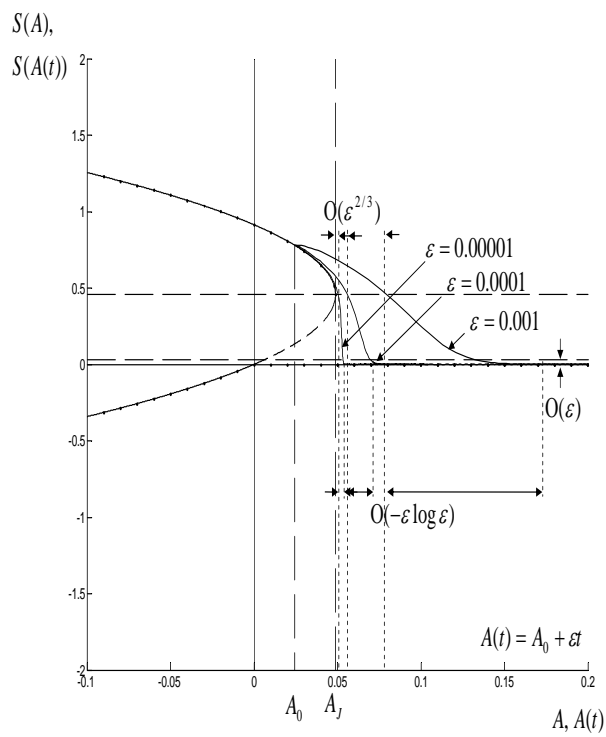

Figure 4: Numerical solutions of (8)-(9) with $A(t)=A_{0}+\epsilon t$ and $A_{0}=\frac{A_{J}}{2}$. We take two different values of $\epsilon$ : (i) $\epsilon=0.001$ and (ii) $\epsilon=0.0001$ and initial condition, $S_{0}=S_{+}\left(\frac{A_{J}}{2}\right)+5 \epsilon, \stackrel{19}{1}$ both cases. 



\section{RECENT REPORTS}

12/67 Wrinkling in the deflation of elastic bubbles

Aumaitre

Knoche

Cicuta

Vella

12/68 Indentation of ellipsoidal and cylindrical elastic shells

Vella

Ajdari

Vaziri

Boudaoud

12/69 Memory of Recessions

Cross

McNamara

Pokrovskiif

12/70 An estimate of energy dissipation due to soil-moisture hysteresis

McNamara

12/71 The Mathematics Behind Sherlock Holmes: A Game of Shadows

Goriely

Moulton

12/72 Some observations on weighted GMRES

Güttel

Pestana

12/73 Bounds on the solution of a Cauchy-type problem involving a Furati weighted sequential fractional derivative

12/74 Static and dynamic stability results for a class of three- Majumdar dimensional configurations of Kirchhoff elastic rods Goriely

12/75 Error estimation and adaptivity for incompressible, nonlinear (hy- Whiteley per)elasticity

Tavener

12/76 A note on heat and mass transfer from a sphere in Stokes flow at Bell low Péclet number

Byrne Whiteley

Waters

12/77 Effect of disjoining pressure in a thin film equation with nonuniform forcing

Moulton

Lega

12/78 A Review of Mathematical Models for the Formation of Vascular Networks

Scianna

Bell

Preziosi

12/79 Fast and Accurate Computation of Gauss-Legendre and Gauss-

Hale Jacobi Quadrature Nodes and Weights

Townsend

$12 / 80$ On the spectral distribution of kernel matrices related to radial

Wathen basis functions

Zhu

12/81 Inner product computation for sparse iterative solvers on dis- Zhu tributed supercomputer 
12/82 A new pathway for the re-equilibration of micellar surfactant solutions

Griffiths

Breward

Colegate

Dellar

Howell

Bain

12/83 Object-Oriented Paradigms for Modelling Vascular Tumour Growth: a Case Study

Connor

Cooper

Byrne

Maini

McKeever

12/84 Chaste: an open source $\mathrm{C}++$ library for computational physiology and biology

Mirams

Arthurs

Bernabeu

Bordas

Cooper

Corrias

Davit

Dunn

Fletcher

Harvey

Marsh

Osborne

Pathmanathan

Pitt-Francis

Southern

Zemzemi

Gavaghan

12/85 A two-pressure model for slightly compressible single phase flow in bi-structured porous media

Schlackow

Marguerat

Proudfoot

Bähler

Erban

Gullerova

12/86 Boolean modelling reveals new regulatory connections between

Lovrics transcription factors orchestrating the development of the ventral spinal cord

Gao

Juhász

Bock

Byrne

Dinnyés

Kovács

12/87 Asymptotic solutions of glass temperature profiles during steady optical fibre drawing

Taroni

Breward

Cummings

Griffiths

12/88 The kinetics of surfactant desorption at the airsolution interface

Morgan

Breward

Griffiths

Howell

Penfold

Thomas

Tucker 
Copies of these, and any other OCCAM reports can be obtained from:

Oxford Centre for Collaborative Applied Mathematics Mathematical Institute

24 - 29 St Giles'

Oxford

OX1 3LB

England

www.maths.ox.ac.uk/occam 